\title{
Evaluación de estudiantes de posgrado en ciencias de la salud
}

\section{Evaluation of post graduate students in health sciences}

\author{
Yury Arenis Olarte, Análida Elizabeth Pinilla \\ - Bogotá, D.C. (Colombia)
}

\section{Resumen}

Este artículo visibiliza a manera de metasíntesis estudios y alcances pedagógicos alrededor de la evaluación del estudiante de posgrado. Inicia contextualizando los cambios paradigmáticos que ha tenido la evaluación en los diferentes niveles de la educación, centrándose en la educación superior, más exactamente en los procesos de enseñanza/evaluación/aprendizaje en ciencias de la salud. Se amplía la visión epistemológica, axiológica y metodológica de la evaluación, de tal manera que se entienda como un proceso complejo, contextualizado, integral y multirrelacional de competencias profesionales con un fin diagnóstico, formativo, de retroalimentación y autorregulación para el mejoramiento.

A nivel de posgrado, la literatura evidencia que los procesos de enseñanza-aprendizaje e investigación se deben orientar al desarrollo de competencias profesionales, a su vez, el proceso de evaluación se articula en éstos según los nuevos enfoques pedagógicos, así, la evaluación es soporte para el aprendizaje y la formación integral de un estudiante de posgrado. En los programas de posgrado en ciencias de la salud, las competencias profesionales incluyen las genéricas o transversales y las específicas, las cuales tienen dimensiones que se desarrollan de forma progresiva. Según la pirámide de Miller, un estudiante para desarrollar una competencia avanza a través de sus diferentes dimensiones: saber-conocer, saber-hacer o saber-cómo, saber-emprender y saber-ser. Entonces, es indispensable contemplar la pirámide de cada competencia profesional, actualizar el concepto de evaluación como proceso eje para el aprendizaje y la enseñanza, todos estos enmarcados en un modelo pedagógico que oriente el enfoque del currículo el cual defina lo qué es evaluación, qué se debe evaluar, cómo evaluar y para qué evaluar.

Profundizando más en la complejidad de la evaluación, es necesario entender cómo un instrumento debe estar adecuado a una dimensión o a las diversas dimensiones a evaluar de una competencia profesional; en programas de posgrado de ciencias de la salud, se pueden utilizar nuevas técnicas e instrumentos de evaluación, tales como: la carpeta de aprendizaje (CA) o portafolio, la evaluación clínica objetiva estructurada (ECOE), las rúbricas, el ejercicio de examen clínico reducido (Mini CEX), entre otras. Por lo anterior, es necesario repensar el paradigma educativo y utilizar los instrumentos pertinentes para las competencias profesionales que se desean evaluar, los cuales deben estar contextualizados y asociados a una situación profesional para tener éxito no sólo en el proceso de evaluación sino en la formación de los futuros egresados. Por tanto, se aportan elementos para que los docentes reflexionen sobre su actuación como evaluadores y, para que la evaluación enriquezca a docentes y discentes en lugar de ocasionarles conflicto en diversos niveles y escenarios. (Acta Med Colomb 2016; 41: 49-57).

Palabras clave: educación basada en competencias, evaluación, aprendizaje, ciencias de la salud.

\section{Abstract}

This article visualizes by metasynthesis studies and pedagogical scopes the assessment of postgraduate students. It starts contextualizing the paradigmatic changes that the evaluation has taken at different levels of education, focusing on higher education, more precisely in the teaching

Dra. Yury Arenis Olarte Arias: Terapeuta Ocupacional. MSc. en Educación. Universidad Nacional de Colombia; Dra. Análida Elizabeth Pinilla Roa. MD. Esp. Internista. Formación en Nutrición y Diabetes Mellitus. Ph.D en Educación. MSc. en Educación con Énfasis en Docencia Universitaria. Especialista en Evaluación y Construcción de Indicadores de Gestión para la Educación Superior. Coordinadora Grupo de Apoyo Pedagógico y Formación Docente. Profesora Asociada. Departamento de Medicina Interna. Facultad de Medicina. Universidad Nacional de Colombia. Bogotá, D.C. (Colombia). Correspondencia. Dra. Análida Pinilla. Bogotá, D.C. (Colombia).

E-mail: yaolartea@yahoo.es

E-mail: aepinillar@unal.edu.co

Recibido: 09/IV/2015 Aceptado: 18/II/2016 
/ evaluation / learning in health sciences. Epistemological, axiological and methodological view of the evaluation is extended, so that it is understood as a complex process, contextualized, comprehensive and multi-relational of professional competences with a diagnosis purpose, educational, of feedback and self-regulation for its improvement.

At postgraduate level, the literature shows that the processes of teaching - learning and research should be directed to the development of professional competences, and in turn, the evaluation process is structured in them according to new pedagogical approaches, so that the evaluation is support for the learning and the integral formation of a postgraduate student. In postgraduate programs in health sciences, professional competences include generic or transversal and specific competences, which have dimensions that are developed in a progressive way. According to Miller's pyramid, for developing a competence, a student advances through different dimensions: know how to recognize, know how to do or know how, know undertake and know to be. So, it is essential to consider the pyramid of each professional competence, updating the concept of evaluation as an axis process for learning and teaching, all these framed in a pedagogical model to guide the focus of the curriculum which define what is evaluation, what should be assessed, how to assess and what to assess.

Deepening more in the complexity of the evaluation, it is necessary to understand how an instrument should be appropriate to a dimension or different dimensions to assess professional competence; in postgraduate programs of health sciences, new techniques and evaluation instruments such as: learning portfolio or portfolio, objective structured clinical evaluation (OSCE), the rubrics, exercise reduced clinical examination (Mini CEX), among others, can be used. Therefore, it is necessary to rethink the educational paradigm and use relevant tools for the desired professional competences that are going to be evaluated, which must be contextualized and linked to a professional situation to succeed not only in the evaluation process but in the formation of future graduates. Therefore, elements are provided for teachers to think on their role as evaluators so that the evaluation may enrich teachers and students rather than cause them conflict at various levels and stages. (Acta Med

Colomb 2016; 41: 49-57).

Keywords: competency-based education, evaluation, learning, health sciences.

\section{Introducción}

Este artículo tiene como propósito sensibilizar e invitar a docentes y estudiantes de posgrado a reflexionar y renovar procesos educativos y en especial la evaluación, desde su acepción paradigmática, metodológica y axiológica. Esto con el fin de optimizar la formación profesional integral que abarca las diversas competencias profesionales (CP) como las específicas (propias de cada profesión o disciplina) y las transversales o comunes éticas, administrativas, pedagógicas y educativas, comunicativas e investigativas, de tal forma que se comprenda por qué es prioritario redimensionar la evaluación como un proceso dinámico, flexible, participativo, concertado; además, diagnóstico (de entrada), formativo y sumativo o terminal por medio del cual un docente brinda apoyo a un estudiante, quien es un profesional en formación posgraduada. Históricamente la evaluación ha acompañado al ser humano, en la vida cotidiana, al generar juicios de valor respecto a algo o alguien; es un fenómeno innato individual y social, por esto se constituye en eje fundamental en educación que gira alrededor de los procesos de enseñanza/ evaluación/aprendizaje.

\section{Antecedentes y evolución de la evaluación}

El concepto de evaluación es un término de transformación contextual que se ha contemplado desde diferentes enfoques y teóricamente ha evolucionado desde la concep- ción propuesta por Tyler (1911) de evaluación por objetivos y de la percepción de rendimiento académico de Fayol que permeaba la educación bajo una visión de producción industrial (1). A partir de esta renovación paradigmática de modelos, se modificó el concepto de evaluación desde la pedagogía, llegando a entenderse como un proceso permanente, formativo, individualizado, contextualizado e integral, visión que en la actualidad abarca no sólo un análisis cuantitativo del evaluado sino cualitativo desde actitudes, habilidades y conocimientos integradas en competencias (2). Actualmente, se concibe la evaluación como un proceso de autorregulación y autogestión del aprendizaje para el mejoramiento continuo de los actores en educación superior, programas e instituciones. Comprendida así, se rescata el sentido complejo, multidireccional y multirrelacional de la evaluación, ya que es un proceso acordado entre estudiantes, profesores y directivos; así mismo, alejado del nepotismo, la improvisación, el poder punitivo y con un fin formativo, de retroalimentación y regulación; por tanto, la función del docente como evaluador ha de ser ejercida con conciencia y responsabilidad para que sea motor y soporte para el progreso de cada actor (3). Sin embargo, los profesores universitarios continúan concibiéndola como el acto de "calificar" luego de aplicar pruebas escritas u orales, técnicas e instrumentos de evaluación que son empleados con el objeto de fijar una nota que puede acreditar 
o no unos "saberes", al terminar un periodo, una asignatura, un semestre; ya que la praxis educativa está subyugada por los procesos tradicionales de formación con un modelo pedagógico enciclopedista, por contenidos, en donde lo que importa es que el estudiante recite un cúmulo de datos y teoría; a pesar de esto, actualmente algunos docentes son conscientes del valor real de la evaluación y aprovechan diversos momentos, metodologías y enfoques de ésta.

La evaluación es un proceso de amplio interés investigativo, por ello diferentes pensadores, pedagogos e intelectuales en distintas épocas de la historia han reconstruido las bases epistemológicas, axiológicas y metodológicas que permiten concebir el evaluar de forma compleja e integral dependiendo del contexto. Así, este tema ha sido motivo de reflexión en todos los niveles de la educación, teniendo auge en la educación básica y media y, un despertar prometedor en la educación superior, con algunas semillas de cambio en la educación universitaria en ciencias de la salud (CS); efectivamente, en el campo pedagógico general es un tema estudiado, pero en programas de posgrado es muy poco lo que se ha desarrollado sobre la evaluación del aprendizaje, mucho menos sobre la evaluación del estudiante y, qué decir, de la evaluación de CP.

Contextualizando, la educación posgradual se desarrolló en Alemania y fue un movimiento importante para la educación durante el siglo XVIII. Esta emergió por influencia de la Ilustración y del proceso de industrialización, de la llamada universidad moderna, la cual adoptó como su esencia la investigación científica, la libertad académica (de enseñar y aprender) y el doctorado en filosofía, como su expresión más alta de conocimiento (4). En Colombia, la formación en posgrados se inició desde mediados del siglo $\mathrm{XX}$ en diversas áreas del conocimiento. Históricamente se muestra un surgimiento desde las especializaciones y especialidades profesionales como las medicoquirúrgicas, más que a programas de maestría y doctorado. Este tipo de formación presenta, por ejemplo los primeros antecedentes formales e institucionales en la década de los 70, con la aprobación mediante el Acuerdo 46 del 9 de mayo de 1978, de la Maestría y el Doctorado en Filosofía de la Universidad Nacional de Colombia, iniciativa pedagógicae investigativa de formación educativa que ha crecido y ha mantenido una participación estable dentro de la educación superior $(5,6)$; en CS cabe mencionar el nacimiento de las especialidades medicoquirúrgicas por esta misma época.

Ahora bien, si se entiende que el nivel posgradual de formación conforma especializaciones, especialidades, maestrías, doctorados y posdoctorados, como programas de alta formación académica e investigativa del núcleo universitario, se afirma que en este nivel educativo están los sujetos en formación con el potencial profesional para construir conocimiento, ciencia y tecnología para el desarrollo de la sociedad y la transformación cultural; incluso como misión posgradual, el estudiante debe ser el principal generador de investigación en su área de conocimiento y desde las necesidades contextuales que le apremian; de esta manera se logra entender la fortaleza pedagógica que se arraiga al integrar los procesos de enseñanza/evaluación/ aprendizaje en la universidad, puesto que en este nivel académico de posgrado no sólo se encuentra el docente como posible sujeto crítico y reflexivo del ejercicio pedagógico, sino que el estudiante es partícipe de procesos para reconstruir y generar conocimientosque aborden situaciones y den soluciones a problemas complejos, interdisciplinarios y multidimensionales de una región o un país.

\section{Algunas investigaciones en evaluación en posgrado}

Este artículo intenta visibilizar a manera de metasíntesis, estudios y alcances pedagógicos de diferentes intelectuales inmersos en programas de posgrado, que han construido conocimiento alrededor del tema de educación, más exactamente en evaluación desde la perspectiva del estudiante para su aprendizaje. Inicialmente se encuentran estudios que abordan la educación y la evaluación en diferentes áreas del conocimiento, generando un análisis pedagógico que incluye la heterogeneidad de la formación en posgrado.

En la investigación realizada por Cabrales (2010) se percibe la evaluación del aprendizaje en los programas de posgrado como un espacio que permite el desarrollo de una evaluación formativa y como una estrategia útil para retroalimentar el aprendizaje, ya que el estudiante es un profesional que se inscribe por vocación e interés y puede como sujeto de formación, contribuir con aspectos claves de análisis para comprender y mejorar la educación en este nivel y, en concreto, el proceso evaluativo que afecta el desempeño profesional y fortalece la autonomía (7). A partir de esta concepción formativa de la evaluación, ésta se concibe como un proceso participativo que puede ser individual o grupal para la retroalimentación, autorregulación y reflexión en torno a ésta (metaevaluación) con la participación del estudiante en la construcción de los criterios de evaluación mediante diálogo y consenso con los profesores, así mismo, la evaluación relaciona el campo profesional y el académico del estudiante y permite la autoevaluación entendida como un medio de autorreflexión, autocontrol, autorregulación y autogestión para el progreso del profesional que continúa formándose y la coevaluación que realiza un condiscípulo; con este enfoque se rescata el ejercicio de la autonomía universitaria (3).

La educación en posgrado es diversa, compleja y precisa de la investigación como sentido fundamental de su existencia $(4,7)$. Por tanto, la evaluación formativa, en este nivel educativo, regula los procesos de enseñanzaaprendizaje e investigación al adaptar y ajustar las condiciones pedagógicas y la jerarquía de los conceptos al contexto metodológico de cada institución y la normatividad. Por ello, se evidencia la necesidad de vincular la evaluación del estudiante en diferentes momentos, procesos y CP, generando una inclusión del campo académico con 
la realidad personal, social y profesional del estudiante, a través del constante acompañamiento docente en diferentes actividades, por ejemplo, en el ejercicio profesional mediante los convenios de docencia-servicio (8). Así, la evaluación formativa, por proceso, se constituye como un factor potencial de la interdisciplinariedad y requiere de la contratación de docentes de tiempo completo con capacitación pedagógica y disciplinar, para crear lazos fuertes con los estudiantes y con el proceso educativo. En suma, la evaluación formativa es crucial para mejorar el servicio que se brinda a un paciente-sujeto, con el fin de llegar a dar una solución a los problemas por los que acude en búsqueda de ayuda profesional.

De otra parte, es necesario resaltar que la educación en posgrado es para profesionales que están en un proceso de formación avanzada y, por ende, en la medida que se avanza en la gama de nivel del posgrado, el estudiante es, cada vez, más autónomo, con un grado importante de motivación, con claridad acerca de su proyecto de vida y metas por superar. En este sentido, este profesional es consciente, crítico y reflexivo, en palabras de Shön (1987) es un "profesional que reflexiona desde y sobre la acción" $(9,10)$; por esto, la autoevaluación es un proceso fundamental para el autoaprendizaje, el desarrollo de CP, el autoconocimiento y para la contribución a una evaluación participativa y formativa que mejore la calidad de la educación para el desempeño profesional.

Sumado a lo anterior, Morales y Trianes (2012) exponen que la educación en posgrado debe servir para la formación de profesionales como agentes de cambio social, no sólo en lo concerniente a la creación y gestión de conocimiento, sino también en el ejercicio de una ciudadanía que propenda por una mayor cohesión social, un ejercicio ciudadano responsable, un compromiso con la comunidad y un actuar con valores, guiados desde el conocimiento cientificoacadémico y la práctica profesional desde y en la universidad (11). Esto brinda, al estudiante de posgrado, la dirección necesaria, pero también la oportunidad de elegir, ofreciéndole diversas actividades que lleven a la práctica su autonomía. Además, es necesario reconocer que se requiere tiempo para que este profesional-estudiante se apropie de un rol protagónico en su aprendizaje, dado que él ya tiene la capacidad de contribuir e incluso liderar procesos pedagógicos y profesionales, entre otros. De esta manera y, con respecto a la evaluación de su aprendizaje, los estudiantes de posgrado consideran necesario la evaluación formativa y coinciden paradójicamente al afirmar que la evaluación en muchos de los casos aún está dirigida sólo a evaluar los resultados de los educandos (12). Al mismo tiempo, se evidencia que los procedimientos de evaluación en posgrado son entendidos desde la acreditación, otorgando un papel pasivo a la educación con sus agentes; entonces, la evaluación desde la acepción de acreditación se concibe para verificar y controlar la calidad del desempeño, a partir del grado de cumplimiento de lo normatizado, "acordado o estableci- do", no como es debido, desde un papel más dinámico e influyente de la retroalimentación, que promueva un cambio paradigmático y contribuya en forma más activa a la calidad de la educación en posgrado (13). Como forma de lograr esa transformación de la evaluación usual, al estudiante de posgrado, hacia una evaluación formativa, Cabrales (2010) planteó cuatro categorías de exploración y análisis para la evaluación formativa desde la percepción de estudiantes: 1. La percepción del estudiante de la función formativa de la evaluación, 2. La percepción del estudiante sobre su participación en el proceso de evaluación, 3 . El manejo de conflictos derivados de la evaluación y 4 . Sugerencias para mejorar los procesos de evaluación (7).

\section{La evaluación de estudiantes en posgrado}

Se aclara, que al rastrear la literatura sobre evaluación del estudiante, en las CS, los estudios se centran en el desempeño del profesional, porque la formación en posgrado está enfocada en su mayoría a la atención de pacientes y, por esto, hay un énfasis en la evaluación de CPclínicas; por tanto, la función social del profesor universitario y de los estudiantes, es generar soluciones a problemas de una persona, una comunidad y una sociedad (14). Así, en el momento actual, se demanda a los actores educativos responsables de los procesos enseñanza/evaluación/aprendizaje la apropiación de un modelo de formación y evaluación de CP.

Se hace preciso entonces hacer un breve recorrido teórico por el concepto de competencia y competencia profesional para entender la evaluación de CP, por ejemplo, clínicas. Según la Real Academia Española el concepto competencia se entiende desde dos acepciones; la primera, competir: es la disputa o contienda entre dos o más personas sobre algo, que aspiran a obtener la misma cosa, y la segunda, competente: es la pericia, aptitud o idoneidad para hacer algo o intervenir en un asunto determinado (15).

En el campo educativo el término competencia hace referencia a la formación integral del ciudadano, es la capacidad que desarrolla una persona para desempeñarse y evidenciar que puede resolver un problema particular dado en un contexto específico y cambiante (16). Por su parte Perrenoud (2004) expone que, el concepto de competencia va más allá de la simple y mera adquisición de conocimientos y habilidades, ya que incluye de forma significativa todas las dimensiones del ser humano y da movilidad a conocimientos, habilidades, saberes, información, actitudes, valores y diferentes esquemas cognoscitivos sobre situaciones análogas para poder afrontarlas y dar posibles soluciones (17). Por su parte, Brailovsky (2001) complementa estas concepciones al referir que una competencia es un constructo complejo, multifacético, multivariado, multidimensional, que a menudo se relaciona con una situación multidisciplinar (18).

Desde la concepción de competencias se especifica, además, que el término competencia profesional está relacionado con la educación universitaria y surgió desde la Organización Internacional del Trabajo (OIT) para rela- 
cionarel sistema educativo con el productivo y la sociedad (Mertens, 1996) (19). Sin embargo, ahora se plantea el concepto de $\mathrm{CP}$, desde un enfoque socioconstructivista como la capacidad que tiene un profesional para utilizar su juicio al integrar conocimientos, habilidades y actitudes para solucionar los problemas complejos que se le presentan en su actividad profesional $(2,18)$. Para comprender el conjunto de las CP se pueden clasificar en 1. Genéricas o transversales y 2. Específicas. Las primeras son comunes a diferentes profesiones: comunicativas, éticas o profesionalismo -la competencia ética es el profesionalismo entendido como un conjunto de valores, principios y normas de actuación con las cuales se ha de ejercer una profesión (20), de gestión y administración, pedagógicas y en educación y, en investigación. Las segundas, son las capacidades especializadas para realizar tareas concretas propias de cada profesión y se aplican en determinado contexto laboral, aquí encontramos en el caso de las CS las competencias clínicas para el cuidado del paciente $(2,21,22)$. De esta manera, un profesional competente o idóneo integra sus CP para aplicarlas a una situación concreta al crear soluciones inéditas, oportunas, particulares y pertinentes frente a problemas que encuentre, por ejemplo, en un paciente; siempre, sin olvidar que este profesional ha de ser cauteloso y reflexivo para que sus intereses y propósitos de vida nunca estén por encima de las necesidades del paciente $(2,21)$.

De acuerdo con lo anterior, se entiende que el concepto de una competencia profesional es un constructo complejo que es accesible de manera parcial, a través de indicadores de los diversos aspectos que se pueden evaluar como los conocimientos, las habilidades y las actitudes de cada CP. Entonces, la mayor parte de los instrumentos que se utilizan actualmente para evaluar una CP no permiten más que una visión parcial; por esto, es necesario repensar el paradigma de la evaluación y utilizar los instrumentos pertinentes a los aspectos y los indicadores de las CP que se desean evaluar, elementos que deben estar siempre contextualizados y asociados a una situación profesional para tener éxito en este proceso de evaluación. Por consiguiente, es indispensable contemplar aparte de la gama de $\mathrm{CP}$ y el porqué de la evaluación de un estudiante, el enfoque del aprendizaje y la enseñanza y renovar el concepto de la evaluación desde: la evaluación de contenidos a la de CP, la evaluación terminal por resultados a la evaluación como proceso para el mejoramiento, la evaluación del aprendizaje a la que lo orienta, es decir, la evaluación para el aprendizaje. Todo lo anterior, enmarcado en una visión socioconstructivista del currículo.

En consecuencia, los programas universitarios de posgrado en CS deben facilitar a los estudiantes el desarrollo de las dimensiones de todas las CP: saber conocer (dimensión cognitiva, conocimientos, conceptos), saber hacer o saber cómo (habilidades mentales y procedimentales, dimensión motora, hacer sabiendo o entendiendo lo que se hace), $s a$ ber emprender (habilidades propositivas, metacognitivas y reflexivas del hacer en contexto) y saber ser (actitudes, valores o virtudes, dimensión afectiva). (21-23), para ser evaluadas de forma permanente, ya que desarrollar CP involucra avanzar y apropiar, cada vez más, las dimensiones de una competencia durante la formación y la vida profesional (Figura 1).

Las nuevas tendencias pedagógicas y evaluativas en educación superior, han pasado indiscutiblemente de un currículo de tipo tradicional hacia uno socioconstructivista para el desarrollo de CP, por ello se expresa la necesidad de encontrar nuevas estrategias didácticas y de evaluación adecuadas hacia este nuevo modelo pedagógico; además,

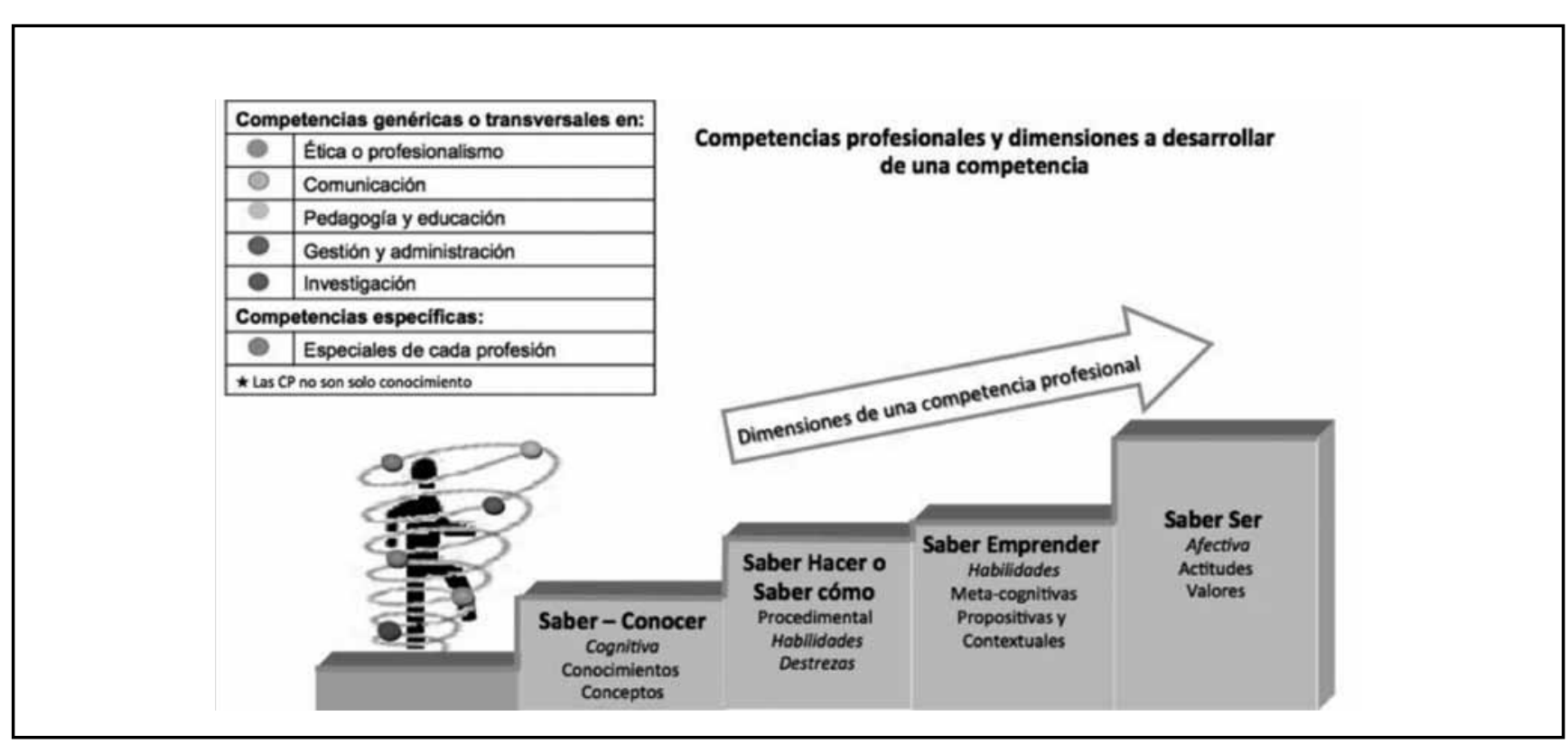

Figura 1. Dimensiones de una competencia profesional. 
se sabe que la evaluación puede generar conflicto entre estudiantes y docentes de diversos niveles, como es patente en la educación superior; lo anterior se da puesto que los encargados de enseñar y evaluar son profesionales de sus respectivas áreas disciplinares que tienen poca o nula preparación en lo que se refiere a la docencia universitaria y, a su vez, la evaluación se complejiza incluso más, cuando se pretende evaluar el desempeño de un estudiante o residente en las actividades clínicas; en muchas ocasiones se les evalúa utilizando los mismos instrumentos de hace años o con cambios pobres con la finalidad de acercarlos a la evaluación de CP, sin un soporte conceptual y colegiado sumado a un cambio organizado, estructurado y ligado a la implementación de este nuevo modelo pedagógico y, por tanto, reforma curricular $(1,2,21)$.

\section{Investigaciones sobre evaluación en programas de posgrado de ciencias de la salud}

Al revisar la literatura, sobre evaluación del estudiante en programas de posgrado, se encontraron diversas publicaciones que en su mayoría son revisiones y propuestas teóricas. Sin embargo, son escasas las investigaciones sobre este tema, no obstante, son un inicio que avizora un impacto en la educación en CS. Diversos investigadores, profesionales del área de la salud y pedagogos se han interesado en el campo de la educación universitaria, más específicamente en el reto de la evaluación, en especial, en la evaluación por CP y, de éstas, en la evaluación de las CP clínicas.

Para llegar a comprender la evaluación de CP, Brailovsky (2001) propone como punto de partida algunas preguntas que posibilitarían el desarrollo sistemático de conceptos pertinentes para este proceso de evaluación: ¿Qué evaluar?, ¿Quién evalúa?, ¿Cómo se evalúa?, ¿Cuándo se evalúa?, ¿Para quién se evalúa?, ¿Por qué hay que evaluar una competencia?, ¿Qué preguntas hay que hacer cuando se trata de evaluar las CP a un estudiante de posgrado? y ¿Dónde se está actualmente respecto de la evaluación de las CP? (18). Ante esta diversidad de cuestionamientos, convergen intelectuales, muchos de ellos médicos, al intentar dar respuesta y aplicar la teoría de la pirámide de Miller (1990) para la evaluación de competencias (24). Sin embargo, la dificultad se evidencia ante las preguntas ¿Cómo se avanza en la pirámide? y ¿Cómo la utilización de los instrumentos de medida favorecen el ascenso en esta pirámide? Aquí, se cuestiona cómo un instrumento organizado para evaluar a un estudiante debe responder a un determinado nivel de la pirámide de Miller, en otras palabras, el instrumento debe estar adecuado a la dimensión a evaluar de una o varias $\mathrm{CP}$ y si éste puede abarcar las diversas dimensiones de una competencia. Esta característica metodológica, de elaboración de instrumentos de medición, complejiza el proceso evaluativo, ya que se prescinde en numerosas oportunidades de la opinión y participación de los estudiantes o de los tutores clínicos o profesionales acompañantes de la prác- tica clínica que laboran en instituciones de salud, quienes refieren poseer escasa o nula formación en evaluación. Esto produce un dilema entre los profesionales clínicos encargados de la tutoría de los educandos, el cuerpo docente y los estudiantes, ya que se presenta discrepancia en las pautas de evaluación, percibiéndose ésta como proceso, pero con cierta subjetividad (25).

\section{Instrumentos para evaluar competencias profesionales}

El camino recorrido hasta la fecha, si bien significa un acercamiento a un sistema de evaluación por CP, aún está lejos de ser lo más óptimo, dado que persisten elementos heredados de un sistema educativo anterior, que limitan la apropiación de nuevas tendencias de evaluación. Además, este proceso se hace más complejo al pretender realizar una evaluación integral, permanente y formativa por CP; que decir, de la dificultad presente al asociar y particularizar el proceso a cada área profesional, a las dimensiones de las $\mathrm{CP}$ a evaluar y, a su vez, a individualizar la evaluación a un profesional-estudiante particular para el éxito del proceso educativo. Ahora bien, si se selecciona entre un gran mundo de profesiones e interrogantes y se cuestiona acerca del cómo podemos evaluar a un profesional-estudiante inmerso en un programa de educación de posgrado de CS, nos encontramos ante una incertidumbre con posibilidad amplia de respuesta, según: el qué, el cómo, el cuándo, el quién, el para qué y el por qué evaluar. Por tanto, es necesaria una planificación del proceso de evaluación que contemple y sea congruente con el perfil de formación del estudiante y con las CP que el currículo plantea; además, categorizar las $\mathrm{CP}$ y los aspectos por evaluar según su importancia en los procesos de enseñanza/evaluación/aprendizaje. Teniendo en cuenta ese análisis se deben determinar los instrumentos evaluativos más adecuados para evaluar las CP.

Los instrumentos de evaluación se pueden clasificar según la manera de exponer los problemas y las respuestas: orales o escritos, si valoran la práctica profesional real o unas pruebas expresamente diseñadas: directos o indirectos o si son más adecuados para valorar conocimientos, habilidades o actitudes de forma separada o integrada cuando se busca evaluar CP $(1,26,27)$. En todo caso, cualquier método de evaluación o instrumentos que se escojan deben evaluar el proceso de formación por $\mathrm{CP}$ semejando lo más posible a la práctica real, teniendo en cuenta lo que se quiere del desempeño profesional y abarcando, en lo posible, todas las dimensiones de las competencias -conocimientos, habilidades y actitudes-; esto se logra al escoger varios instrumentos de evaluación que se complementen y que abarquen diferentes $\mathrm{CP}$ en sus diferentes dimensiones o al estructurarlos con una visión integradora.

Ante estos desafíos, se registra en algunas investigaciones, sobre evaluación del estudiante en programas de posgrado de CS, propuestas de nuevas técnicas e instrumentos de evaluación, tales como: la autoevaluación, la 
evaluación por pares (coevaluación), la simulación de procesos de aprendizaje y evaluación, el aprendizaje basado en problemas, la evaluación por carpeta de aprendizaje (CA) o el portafolio, el ensayo, la evaluación clínica objetiva estructurada (ECOE), las rúbricas, el ejercicio de examen clínico reducido (Mini CEX), entre otras. Innovaciones pedagógicas y metodológicas que exaltan compromiso y consenso entre responsables, ya que en este tema no existe una receta única ni un instrumento ideal que concierne a la evaluación de CP.

A continuación se exponen cuatro técnicas o instrumentos de evaluación, cada docente o coordinador de programa puede contextualizar, según los intereses y las necesidades para evaluar $\mathrm{CP}$ a un estudiante de posgrado; se profundiza en técnicas e instrumentos pertinentes para evaluar, en particular, en las dos niveles superiores de la pirámide Miller, es decir, las dimensiones de las CP del demostrar cómo y el desempeño profesional, niveles que se deben desarrollar y fortalecer en la formación de posgrado en médicos y demás profesionales de las CS (Figura 2).

\section{Portafolio o carpeta de aprendizaje}

La carpeta de aprendizaje (CA) se concibe como un instrumento que permite tanto la organización del aprendizaje del estudiante durante el proceso, como la evaluación del mismo. Es una técnica formativa y evaluativa basada en un conjunto de evidencias que elabora el estudiante respecto al aprendizaje adquirido, éste debe acreditar todo conocimiento que afirma, reflexionando sobre el proceso formativo que ha llevado a cabo, generando un tipo de evaluación continuada, procesual, compleja, multidimensional, orientada a la retroacción, contextualizada, personalizada y colaborativa $(26,27)$. La CA, se concibe como una herramienta evaluativa formativa en donde la evaluación es un proceso y no un suceso aislado, en donde siempre es un medio y nunca un fin. Este instrumento constituye una forma de valoración de las CP en el posgrado, por su carácter creativo, innovador, participativo y dinámico, que refleja quién es el estudiante, su trabajo en colaboración, la relación con el paciente, el trabajo en grupos, la resolución de problemas contextuales y la reflexión de procesos, en fin la evaluación basada en el desempeño. Entonces la CA, es una manera adecuada de evaluación integral que analiza los conocimientos, las habilidades y las actitudes de los estudiantes, es un trabajo documental a través de un proceso de reflexión de experiencias, para establecer un puente entre lo que se sabe, lo que se está aprendiendo y el desempeño real o auténtico. Esto se logra desde la identificación de experiencias significativas positivas y negativas con fundamento pedagógico sólido, que se registran con un sentido constructivo alejado de un registro frío y mecánico.

La evaluación por CA debe incluir las áreas de conocimiento o asignaturas que den cuenta de $\mathrm{CP}$ integrales del profesional-estudiante con contenidos conceptuales, procedimentales y actitudinales, visualizados a través de la reflexión crítica. Se debe tener presente siempre el espacio o contexto donde se hace la práctica académica/profesional y escoger los instrumentos adecuados de evaluación, manteniendo una relación estrecha con las competencias a evaluar para no fracasar.

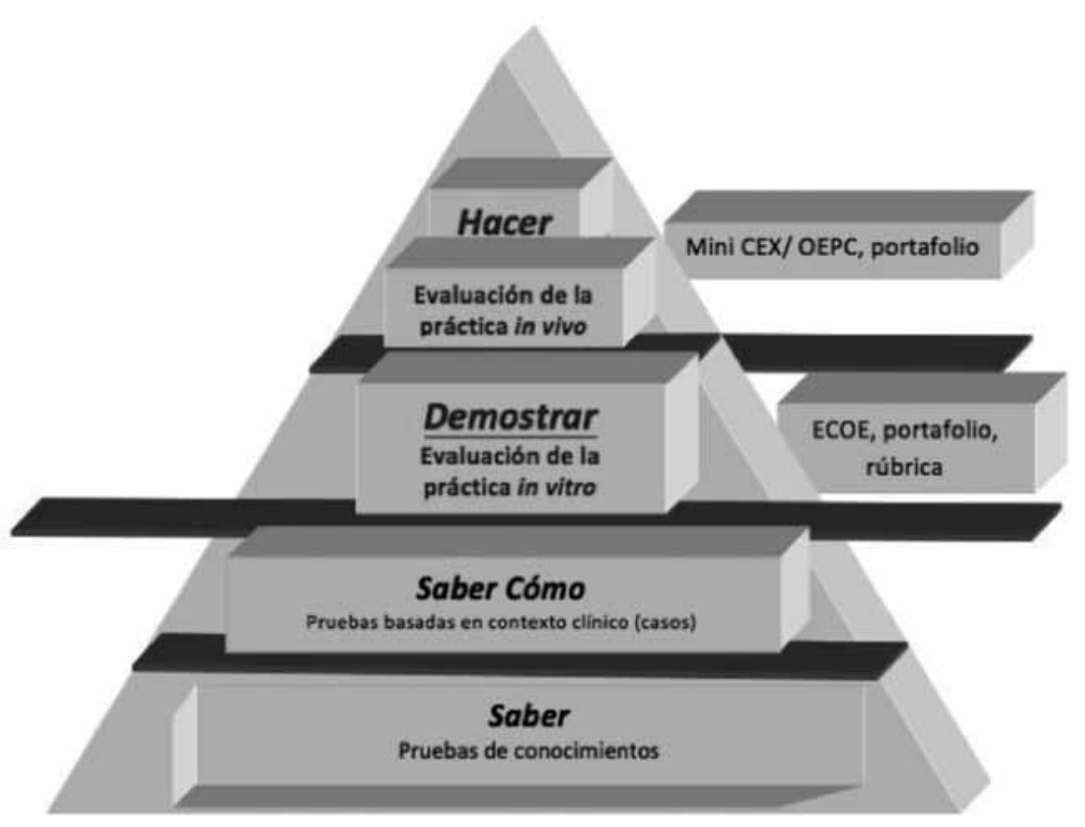

Figura 2. Relación pirámide de Miller e instrumentos de evaluación según nivel de desarrollo de una competencia profesional. 


\section{Evaluación clínica objetiva estructurada (ECOE)}

La ECOE es un formato de examen que incorpora diversos instrumentos evaluativos y se desarrolla a lo largo de diferentes estaciones que simulan situaciones clínicas. La potencia de este formato radica en la mezcla de métodos de evaluación, de manera que es capaz de explorar casi todos los niveles de la pirámide de Miller: saber, saber cómo y demostrar cómo (28). Para la realización del ECOE es necesario un comité de prueba, el cual es un elemento fundamental, ya que es el órgano colegiado responsable de los contenidos de la misma. Este comité está constituido por un conjunto de profesionales clínicos de reconocida experiencia y se encarga de estipular los componentes de las competencias del profesional-estudiante, de definir los criterios e indicadores de ponderación de cada CP y de elaborar el conjunto de situaciones clínicas y profesionales con las que se va a evaluar. Estas situaciones clínicas se explicitan en casos, los cuales se deben asemejar lo más posible a la realidad y deben posibilitar la evaluación de uno o varios de los componentes de las CP. A partir de este proceso se elabora la tabla de especificaciones, la cual es el documento base de una ECOE. Esta tabla representa el diseño general de la prueba, constituida por una parrilla de filas y columnas, de manera que se relacionan los casos, con los instrumentos evaluativos que se utilizan y con los criterios e indicadores a evaluar. Cada una de las filas corresponde a una situación clínica, de manera que habrá tantas como estaciones compongan la ECOE.

Cabe señalar que el profesional-estudiante que realiza una ECOE sabe en muchas ocasiones que le va a ser evaluado, pero desconoce qué de éstas se le está evaluando en cada estación. De esta manera, en todas las situaciones clínicas el estudiante debe actuar de la manera más profesional posible, ya que a diferencia de otras pruebas más simples y directas no se trata sólo de acertar el diagnóstico o de prescribir el tratamiento adecuado, sino de ejercer como profesional idóneo o competente en todos los casos, poniendo en juego de forma orquestada sus conocimientos, habilidades y actitudes $(26,28,29)$.

\section{Rúbricas}

La rúbrica o matriz de valoración es una estrategia de evaluación generada a través de un listado de criterios específicos y fundamentales que promueven el aprendizaje de los estudiantes para el desarrollo de competencias. Este instrumento de evaluación es una guía para fomentar el aprendizaje por su carácter retroalimentador, formativo y autoevaluativo que ayuda a dirigir el nivel de progreso de los estudiantes. Con la rúbrica se busca que los estudiantes a través del uso, mejoren o adquieran gradualmente diversas $\mathrm{CP}$ en su dimensión conceptual, procedimental y actitudinal. A través de la rúbricas los profesionales-estudiantes logran hacer explícito lo tácito, de ahí que la identificación de los componentes o criterios e indicadores de las CP se consideren como fundamentales para el desarrollo de un estudiante.

Por lo general las rúbricas se conocen como rúbricas comprensivas (holistas) y rúbricas analíticas; las primeras, contienen valoraciones generales que no involucran necesariamente un listado de niveles de ejecución o rubros (contenidos) y, las segundas, involucran respuestas muy bien enfocadas a una serie de conceptos, junto con la escala de evaluación correspondiente, definiendo cada uno de sus descriptores. Usualmente las rúbricas constan de tres componentes: 1) conceptos o rubros) 2) escala de calificación (o nivel de ejecución) y 3 ) criterios o descriptores $(30,31)$.

\section{Mini CEX}

El Mini Clinical Evaluation Exercise (Mini-CEX), también llamado prueba de Observación Estructurada de la Práctica Clínica (OEPC), se puede definir como un método de observación directa de la práctica profesional con evaluación estructurada mediante un formulario y posterior realización de retroalimentación al profesional-estudiante. El Mini-CEX está indicado para evaluar los componentes de competencias como habilidades de entrevista clínica, habilidades de exploración física, profesionalismo, juicio clínico, habilidades comunicativas, organización, entre otras. Es un instrumento adecuado para las CP clínicas que se basa en casos clínicos con pacientes reales y con retroalimentación inmediata a la culminación de la evaluación. Estos casos clínicos son de tipología y complejidad diferentes y con observadores y entornos distintos. Es un instrumento evaluativo que dispone de una ficha estructurada para la recolección de la información y con tiempo promedio de ejecución de 30 minutos. (Observación y retroalimentación). Por tanto, las características propias del Mini-CEX, le otorgan validez suficiente para ser utilizado tanto en la evaluación formativa como sumativa (32).

\section{Reflexiones finales}

Sin duda alguna se necesita mucha más investigación sobre los efectos educativos de la evaluación. Además, la utilidad de los instrumentos depende del contexto en que se emplean y de las concepciones de los profesores que los utilizan. Para realizar una evaluación pertinente se debe cubrir toda la pirámide de una $\mathrm{CP}$, porque si los evaluadores se limitan a los dos pasos inferiores de la pirámide estarán en el nivel de la evaluación cognitiva de las CP "saber y saber cómo". Por tanto, es importante recordar que sólo se puede evaluar lo que se puede observar durante el desempeño; siempre hay que identificar y definir las metas y las CP que los estudiantes deben alcanzar en una actividad de formación, de igual forma, es indispensable explicitárselas y lograr construir los instrumentos necesarios para poder evaluar de la mejor manera lo que se desea.

Por tanto, los procesos educativos en la educación posgraduada en CS, son complejos por la necesidad pedagógica y evaluativa de contemplar la integralidad de las CP para 
la formación adecuada de profesionales. Por ello, el acto de evaluar de forma pertinente es un reto inminente para docentes, directivos y estudiantes, teniendo en cuenta que aún se usan instrumentos de evaluación vertical y terminal; queda claramente planteado, la proyección paradigmática constructivista hacia una evaluación integral, individual, en espiral, es decir, progresiva, que incluso comienza cuando un estudiante inicia una asignatura o una rotación, en otras palabras, una evaluación diagnóstica que luego sigue hacia una evaluación formativa y culmina con una evaluación final. De esta manera, se podría identificar más rápidamente las dificultades en los procesos de enseñanza/evaluación/ aprendizaje y por tanto, se lograría un desarrollo más óptimo de las CP con un ambiente favorable para el aprendizaje y el desarrollo de éstas.

Por esto, los cursos de posgrado deben promover la investigación y reflexión crítica del conocimiento y del contexto del quehacer profesional y pedagógico de los profesionales estudiantes, para que el aprendizaje se vuelva altamente significativo y promueva el autoaprendizaje en toda la vida. En consecuencia, es prioritario que los docentes se formen como evaluadores para que puedan diseñar nuevos sistemas de evaluación holísticos que reflejen el modelo pedagógico y el currículo, para evidenciar un cambio del formar para evaluar (enseñanza) a evaluar para formar, para orientar y para alcanzar el desarrollo de CP (33).

\section{Referencias}

1. Pinilla AE. Metodología de la evaluación académica. En: Rojas E, Vera L, Madiedo N, editores. Reflex edu univer III. Primera edición. Bogotá, Unibiblos, 2007. p. 165-89.

2. Pinilla AE. Construcción y evaluación de un perfil de competencias profesionales en medicina interna. Cole Desa Hum. Bogotá: Editorial Universidad Nacional de Colombia; 2015.

3. Pinilla AE. Hacia el nuevo paradigma de la autoevaluación: el mejoramiento social. En: Madiedo N; Pinilla AE; Sánchez J, editores. Reflex edu univer II: Eval. Segunda edición. Bogotá: Editorial Facultad de Medicina, Universidad Nacional de Colombia. 2008. p. 163-72.

4. Morales V. La universidad latinoamericana actual: Necesidad de replantear su misión. Rev Pedag. 1999. p. 1-10 Citado el 27 de julio de 2015. Disponible en: http://www.saber.ula.ve/bitstream/123456789/22144/1/articulo9.pdf

5. Universidad Nacional de Colombia. Claves para el debate público. Posgrado en Colombia y América Latina: Nivel académico que contribuye al desarrollo del conocimiento. Unimedios. 2009; 25: 6-13. Citado el 27 de julio de 2015. Disponible en: http://www.agenciadenoticias.unal.edu.co/uploads/media/ Claves_Digital_No._05.pdf

6. Jaramillo H. La formación de posgrado en Colombia: maestrías y doctorados. Rev Iberoameri Cienc Tecno Soci - CTS. 2009; 13 (5): 131-155. Citado el 27 de julio de 2015. Disponible en: http://http://www.revistacts.net/volumen-5-numero-13.

7. Cabrales O. Evaluación de los aprendizajes en los estudios de postgrado bajo los principios de la evaluación participativa. Rev Edu Desa Soc. 2010; 4 (1): 10-37. Citado el 27 de julio de 2015. Disponible en: http://www.umng.edu.co/documen ts/63968/80126/01Evaluacion.pdf

8. Decreto 2376 de julio 1 de 2010. Por medio del cual se regula la relación docencia-servicio para los programas de formación de talento humano del área de la salud. Ministerio de la Protección Social. Citado el 27 de julio de 2015. Disponible en http://www.ut.edu.co/administrativo

9. Shön, D.A. La enseñanza del arte a través de la reflexión en la acción. En: La formación de profesionales reflexivos. Hacia un nuevo diseño de la enseñanza y el aprendizaje en las profesiones. Madrid: Editorial Paidós. 1987. p 33-48.

10.Shön, D.A. De la racionalidad técnica a la reflexión desde la acción. En: El profesional reflexivo. Barcelona: Paidós. 1998., p 31-73.
11. Morales FM, Trianes MV. Evaluación de actitudes hacia temas morales en una muestra de estudiantes de posgrado. Rev Forma Innov Edu Univ. 2012; 5(2): 88-98. Citado el 27 de julio de 2015. Disponible en: http://webs.uvigo.es/refiedu/Refiedu/ Vol5_2/REFIEDU_5_2_3.pdf

12.Pulido JE. Paradoja del proceso de evaluación de los alumnos de postgrado de la Universidad Pedagógica Experimental Libertador, caso: instituto de mejoramiento profesional magisterio. Invest Postgr. 2007; 22 (2): 87 -114.

13. Granados H, Mercado I, Delgado A. Evaluación académica del posgrado: un estudio de los procedimientos de gestión aplicados en el ámbito latinoamericano. Universidades. 2004; 27: 29-38. Citado el 27 de julio de 2015. Disponible en: http://www.clacso-posgrados.net/documentos_aportes/27.pdf

14.Pinilla AE. Medicina y Educación. Editorial. Rev Facult Med Univ Nacio. 2012; 59: $275-78$.

15.Real Academia Española. [Citado 2012 diciembre 19] Disponible en: www. rae.es

16. Pinilla AE. Recuperando la identidad del médico internista. Al visualizar las competencias profesionales. Acta Med Colomb. 2010; 35: 143-53.

17. Perrenoud P. Diez nuevas competencias para enseñar. Barce Edit Grao. 2004.pp. 88-91.

18.Brailovsky C. Educación Médica: Evaluación de las competencias. En: Aportes para un Cambio Curricular en Argentina 2001. Facultad de Medicina, UBA. 2001 p 103-121 [Citado 2012 diciembre 19] Disponible en: http://publicaciones. ops.org.ar/publicaciones/otras\%20pub/Aportes $\% 20 \mathrm{p}$ ara $\% 20 \mathrm{un} \% 20$ cambio $\% 20$ curricular.pdf

19.Mertens, L. Competencia laboral: sistemas, surgimiento y modelos. Montevideo: Cinterfor. (1996). Extraído el 26 de julio de 2015 desde http://www.oei.es/etp/ competencia laboral sistemas modelos mertens. pdf

20.Escudero JM, Vallejo M, Botías F. El asesoramiento en educación ¿Podrían ser las competencias profesorado una contribución positiva? Rev Curric Forma Profes. 2008; 12: 1-23.

21.Pinilla AE. Modelos pedagógicos y formación de profesionales en el área de la salud. Act méd Colom. 2011; 36 (4): p 204 - 218.

22.Pinilla A.E. Aproximación conceptual a las competencias profesionales en ciencias de la salud. Rev Salud Públ. 2012; 14 (5): 852-64.

23.Pinilla AE. Las competencias en educación universitaria. En: Madiedo N; Pinilla AE; Sánchez J, editores. Reflexiones en educación universitaria II: Evaluación. Segunda edición. Bogotá: Edit Facul Med, Unive Nacio Colom. 2008. p. 98-129.

24. Miller, G. The assessment of clinical skills/competence/ performance. Acade Med. 1990; 65 (9): p 63-67.

25.Marrodán G. Percepción de académicos, supervisores de prácticas clínicas estudiantes y egresados sobre validez del sistema de evaluación de competencias específicas utilizado en la carrera de Obstetricia en la Universidad de Antofagasta. Rev Educ Cienc Salud. 2009; 6 (2): 101-10.

26.Pinilla AE. Evaluación de competencias profesionales en salud. Rev Fac Med Univ Nac Colomb. 2013; 61 (1): 53-70.

27. Arreciado M, Cónsul G. Reflexión y evaluación de las prácticas clínicas del postgrado de enfermería quirúrgica a través de la carpeta de aprendizaje. Rev Docen Univer. 2011; 9 (3): p 181-197.

28.De Serdio, R. ECOE: Evaluación Clínica Objetiva Estructurada. I. Competencias y su evaluación. Med Famil (And) 2002; 3 (1): 49-52. http://www.samfyc.es/ Revista/PDF/v3n1/049-052.pdf

29.De Serdio, R., ECOE: Evaluación Clínica Objetiva Estructurada. Med Famil (And) 2002; 3 (2): 127-32. Citado el 27 de julio de 2015. Disponible en: http:// www.um.es/c/document_library/get_file?uuid=9fa20d68-26f6-430c-84517842598bea $17 \&$ groupId=115466

30.López, MA. Guía Básica para la elaboración de rúbricas. Univ Iberoameri Puebla. 2007. Citado el 27 de julio de 2015. Disponible en: http://es.slideshare.net/alcado/ gua-bsicaparalaelaboracinderbricas 12035582631640313

31.Bujan K, Rekalde I, Aramendi P. La evaluación en la educación superior. Las rúbricas como instrumento de evaluación. Bogotá: Edit MAD S.L. 2011.

32.Fornells-Vallés JM. El ABC del Mini CEX. Educ Med. 2009; 12 (2): 839. Citado el 27 de julio de 2015. Disponible en: http://www.medicina.ufg.br/ uploads/148/original_El_ABC_del_Mini-CEX.pdf

33. Lavalle M. Retos en la educación médica de posgrado: el reto de la evaluación. [Vídeo]. Conferencia segundo congreso nacional en educación médica y IV encuentro de educación médica de la CCINSHAE. Facult Med UNAM Univ Auton México. 2012. Citado el 27 de julio de 2015. Disponible en: https://www. youtube.com/watch?v=_3dErpU0TaE 